\title{
Full-Order State Observer Design for Nonlinear Systems Based on Piecewise Bilinear Models
}

\author{
Tadanari Taniguchi, Luka Eciolaza, and Michio Sugeno
}

\begin{abstract}
This paper proposes a full-order state observer design of nonlinear control systems approximated by piecewise bilinear (PB) models. We construct PB model of nonlinear control systems. The approximated system is found to be fully parametric. The input-output (I/O) feedback linearization is applied to stabilize PB control systems. The design method is capable of designing the state observer and the servo controller of nonlinear systems separately. Although the controller is simpler than the conventional $\mathbf{I} O \mathrm{O}$ feedback linearization controller, the control performance based on PB model is the same as the conventional one. We present the PB modeling combined with the conventional feedback linearization as a very powerful tool for the analysis and synthesis of nonlinear control systems. An illustrative example confirms the feasibility of our proposals.
\end{abstract}

Index Terms-Nonlinear control, piecewise bilinear model, input-output linearization, state observer.

\section{INTRODUCTION}

Piecewise linear (PL) systems which are fully parametric have been intensively studied in connection with nonlinear systems [1]-[4]. We are interested in the parametric piecewise approximation of nonlinear control systems based on the original idea of PL approximation. The PL approximation has general approximation capability for nonlinear functions with a given precision. However, it is difficult to handle some PL system such as simplexes in the rectangular coordinate system.

To overcome this difficulty, one of the authors suggested to use the piecewise bilinear (PB) approximation [5]. We note that a bilinear function as a basis of $\mathrm{PB}$ approximation is, as a nonlinear function, the second simplest one after a linear function. The model has the following features. 1) The PB model is derived from fuzzy if-then rules with singleton consequents. 2) It is built on piecewise hypercubes partitioned in the state space. 3) It has general approximation capability for nonlinear systems. 4) It is a piecewise nonlinear model, the second simplest after a PL model. 5) It is continuous and fully parametric. So far we have shown the necessary and sufficient conditions for the stability of PB systems with respect to Lyapunov functions in the two dimensional case [6], [7] where membership functions are fully taken into account. We derived the stabilizing conditions [8], [9] based on the feedback

Manuscript received December 9, 2013; revised February 11, 2014.

T. Taniguchi is with IT Education Center, Tokai University, Hiratsuka, Kanagawa, 2591292 JAPAN (email: taniguchi@tokai-u.jp).

L. Eciolaza and M. Sugeno are with European Centre for Soft Computing, (e-mail: michio.sugeno@gmail.com). linearization, where [8] applies the input-output linearization and [9]-[10] applies the full-state linearization. In the feedback linearization, we design a state feedback controller which transforms a nonlinear system into an equivalent linear system.

This paper proposes a full-order state observer of PB control system to estimate the coordinate transformation $z$. This design method is capable of designing the state observer and the controller of nonlinear systems separately. The full-order state observer can be designed for all the PB control systems since the feedback linearized system is observable. Although the controller and observer are simpler than the conventional I/O feedback linearization controller, the performance based on $\mathrm{PB}$ model is equivalent to the conventional one.

This paper is organized as follows. Section II presents the canonical form of PB models. Section III presents PB controllers for nonlinear plants with $\mathrm{PB}$ modeling and I/O linearization. Section IV proposes a design method of observer-based PB controller. Section V shows an example demonstrating the feasibility of the proposed methods, and Section VI gives conclusions.

\section{CANONICAL FORM OF PB MODELS}

\section{A. Open-Loop Systems}

In this section, we introduce the PB models suggested in [5]. We deal with the two dimensional case without loss of generality. Define a vector $d(\sigma, \tau)$ and a rectangle $R_{\sigma \tau}$ in the two-dimensional space as, respectively,

$$
\begin{gathered}
d(\sigma, \tau) \equiv\left(d_{1}(\sigma), d_{2}(\tau)\right)^{T} \\
R_{\sigma \tau} \equiv\left[d_{1}(\sigma), d_{1}(\sigma+1)\right] \times\left[d_{2}(\tau), d_{2}(\tau+1)\right]
\end{gathered}
$$

$\begin{array}{cccrr}\sigma \quad \text { and } \tau & \text { are } & \text { integers: } \quad-\infty<\sigma, \tau<\infty & \text { where } \\ d_{1}(\sigma)<d_{1}(\sigma+1) & , & d_{2}(\tau)<d_{2}(\tau+1) & \text { and } \\ d(0,0) \equiv\left(d_{1}(0), d_{2}(0)\right)^{T} . & \text { The } & \text { superscript } & T & \text { denotes }\end{array}$ transpose operation. For $x \in R_{\sigma \tau}$, the $\mathrm{PB}$ system is expressed as

$$
\left\{\begin{array}{l}
\dot{x}=\sum_{i=\sigma}^{\sigma+1} \sum_{j=\tau}^{\tau+1} \omega_{1}^{i}\left(x_{1}\right) \omega_{2}^{j}\left(x_{2}\right) f(i, j), \\
x=\sum_{i=\sigma}^{\sigma+1} \sum_{j=\tau}^{\tau+1} \omega_{1}^{i}\left(x_{1}\right) \omega_{2}^{j}\left(x_{2}\right) d(i, j),
\end{array}\right.
$$

where 


$$
\left\{\begin{array}{l}
\omega_{1}^{\sigma}\left(x_{1}\right)=\left(d_{1}(\sigma+1)-x_{1}\right) /\left(d_{1}(\sigma+1)-d_{1}(\sigma)\right), \\
\omega_{1}^{\sigma+1}\left(x_{1}\right)=\left(x_{1}-d_{1}(\sigma)\right) /\left(d_{1}(\sigma+1)-d_{1}(\sigma)\right), \\
\omega_{2}^{\tau}\left(x_{2}\right)=\left(d_{2}(\tau+1)-x_{2}\right) /\left(d_{2}(\tau+1)-d_{2}(\tau)\right), \\
\omega_{2}^{\tau+1}\left(x_{2}\right)=\left(x_{2}-d_{2}(\tau)\right) /\left(d_{2}(\tau+1)-d_{2}(\tau)\right),
\end{array}\right.
$$

and $\omega_{2}^{j}\left(x_{2}\right) \in[0,1]$. In the above, we assume $f(0,0)=0$ and $d(0,0)=0$ to guarantee $\dot{x}=0$ for $x=0$.

A key point in the system is that the state variable $x$ is also expressed by a convex combination of $d(i, j)$ with respect to $\omega_{1}^{i}\left(x_{1}\right)$ and $\omega_{2}^{j}\left(x_{2}\right)$ just as in the case of $\dot{x}$. As is seen in Eq. (3), $x$ is located inside $R_{\sigma \tau}$ which is a rectangle: a hypercube in general. That is, the expression of $x$ is polytopic with four vertices $d_{1}(\sigma), d_{1}(\sigma+1), d_{2}(\tau)$ and $d_{2}(\tau+1)$. The model of $\dot{x}=f(x)$ is built on a rectangle including $x$ in the state space and it is also poly topic with four vertices $f(\sigma, \tau), f(\sigma+1, \tau), f(\sigma, \tau+1)$ and $f(\sigma+1, \tau+1)$.We call this form of the canonical model (2) parametric expression.

Representing $\dot{x}$ with $x$ in Eqs. (2) and (3), we can obtain the state space expression of the model which is found to be bilinear (bi-affine) [5]. Therefore, the derived PB model has simple nonlinearity. In the case of the PL approximation, a PL model is built on simplexes partitioned in the state space, triangles in the two dimensional case. Note that any three points in the three dimensional space are spanned with an affine plane: $y=a+b x_{1}+c x_{2}$. A PL model is continuous. It is, however, difficult to handle simplexes in the rectangular coordinate system.

Also we can see that any four points in the three dimensional space can be spanned with a bi-affine plane: $y=a+b x_{1}+c x_{2}+d x_{1} x_{2}$. In contract to a PL model, a PB model as such is built on rectangles with the four vertices, on hypercubes in a general dimensional space, partitioned in the state space; it well matches the rectangular coordinate system. Therefore, PB models would be applicable to control purpose.

\section{B. Closed-Loop Systems}

We consider a two-dimensional nonlinear control system.

$$
\left\{\begin{array}{l}
\dot{x}=f_{o}(x)+g_{o}(x) u(x) \\
y=h_{o}(x) .
\end{array}\right.
$$

The PB model (5) can be constructed from the nonlinear system (4).

$$
\left\{\begin{array}{l}
\dot{x}=f(x)+g(x) u(x), \\
y=h(x),
\end{array}\right.
$$

where

$$
\left\{\begin{array}{l}
f(x)=\sum_{i=\sigma}^{\sigma+1} \sum_{j=\tau}^{\tau+1} \omega_{1}^{i}\left(x_{1}\right) \omega_{2}^{j}\left(x_{2}\right) f(i, j), \\
g(x)=\sum_{i=\sigma}^{\sigma+1} \sum_{j=\tau}^{\tau+1} \omega_{1}^{i}\left(x_{1}\right) \omega_{2}^{j}\left(x_{2}\right) g(i, j), \\
h(x)=\sum_{i=\sigma}^{\sigma+1} \sum_{j=\tau}^{\tau+1} \omega_{1}^{i}\left(x_{1}\right) \omega_{2}^{j}\left(x_{2}\right) h(i, j) .
\end{array}\right.
$$

The modeling procedure in the region $R_{\sigma \tau}$ is as follows. Algorithm 2.1: Piecewise bilinear modeling procedure

1) Assign vertices $d(i, j)$ for $x_{1}=d_{1}(\sigma), d_{1}(\sigma+1)$, $x_{2}=d_{2}(\tau), d_{2}(\tau+1)$ of the state vector $x$, then the state space is partitioned into piecewise regions, see also Fig. 1.

2) Compute the vertices $f(i, j), g(i, j)$ and $h(i, j)$ in Eqs. (6), by substituting the values of $x_{1}=d_{1}(\sigma)$, $d_{1}(\sigma+1)$ and $x_{2}=d_{2}(\tau), d_{2}(\tau+1)$ into original nonlinear functions $f_{o}, g_{o}$ and $h_{o}$ in the system (4). Fig. 1 illustrates $f_{1}(x)$ and $x \in R_{\sigma \tau}$.

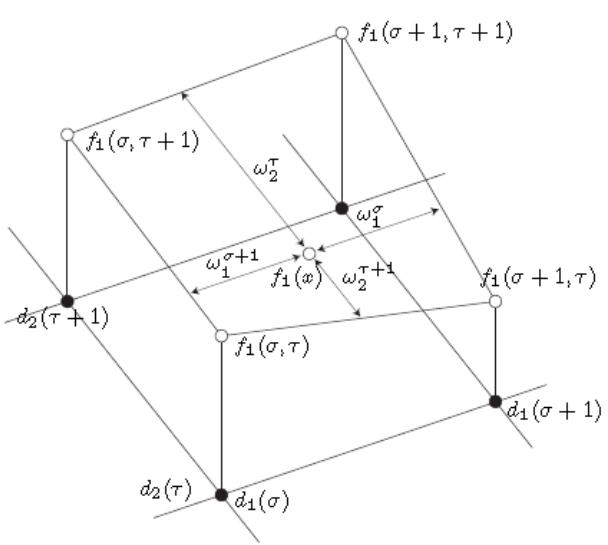

Fig. 1. Piecewise region $\left(f_{1}(x), x \in R_{\sigma \tau}\right)$.

The overall PB model can be obtained automatically when all the vertices are assigned. Note that $f(x), g(x)$ and $h(x)$ in the PB model coincide with those in the original system at the vertices of all the regions.

\section{DESIGN OF PB CONTROLLERS FOR NONLINEAR MODELS WITH PB MODELING AND I/O LINEARIZATION}

This section deals with the I/O linearization of nonlinear control systems approximated with PB models. We consider, in particular, nonlinear systems and show their I/O linearization based on PB models in detail. We also show that in the case of PB systems, the I/O linearization (the feedback linearization in general) may be applicable to a global region by avoiding the restrictions of the conventional linearization of nonlinear control system: the restriction concerning the relative degree. First we give a brief introduction to the I/O linearization of PB models [9], [10]. Due to lack of space, we use $\omega_{1}^{i_{1}}$ and $\omega_{2}^{i_{2}}$ in $\omega_{1}^{i_{1}}\left(x_{1}\right)$ and $\omega_{2}^{i_{2}}\left(x_{2}\right)$ in this section.

\section{A. I/O linearization}

Consider the PB model (5) in the previous section. The derivative $y$ is given by

$$
\dot{y}=\frac{\partial h}{\partial x}(f(x)+g(x) u)=L_{f} h(x)+L_{g} h(x) u,
$$

where 


$$
\begin{aligned}
& L_{f} h(x)=\sum_{i_{2}=\sigma_{2}}^{\sigma_{2}+1} \omega_{2}^{i_{2}} \cdots \sum_{i_{n}=\sigma_{n}}^{\sigma_{n}+1} \omega_{n}^{i_{n}} \frac{h\left(\delta_{1}, i_{2}, \ldots, i_{n}\right)}{d_{1}\left(\delta_{1}\right)} f_{1}+\cdots \\
&+\sum_{i_{1}=\sigma_{1}}^{\sigma_{1}+1} \omega_{1}^{i_{1}} \cdots \sum_{i_{n-1}=\sigma_{n-1}}^{\sigma_{n-1}+1} \omega_{n-1}^{i_{n-1}} \frac{h\left(i_{1}, i_{2}, \ldots, \delta_{n}\right)}{d_{n}\left(\delta_{n}\right)} f_{n}, \\
& L_{g} h(x)= \sum_{i_{2}=\sigma_{2}}^{\sigma_{2}+1} \omega_{2}^{i_{2}} \cdots \sum_{i_{n}=\sigma_{n}}^{\sigma_{n}+1} \omega_{n}^{i_{n}} \frac{h\left(\delta_{1}, i_{2}, \ldots, i_{n}\right)}{d_{1}\left(\delta_{1}\right)} g_{1}+\cdots \\
&+\sum_{i_{1}=\sigma_{1}}^{\sigma_{1}+1} \omega_{1}^{i_{1}} \cdots \sum_{i_{n-1}=\sigma_{n-1}}^{\sigma_{n-1}+1} \omega_{n-1}^{i_{n-1}} \frac{h\left(i_{1}, i_{2}, \ldots, \delta_{n}\right)}{d_{n}\left(\delta_{n}\right)} g_{n}, \\
& h\left(\delta_{1}, i_{2}, \ldots, i_{n}\right)=h\left(\sigma_{1}+1, i_{2}, \ldots, i_{n}\right)-h\left(\sigma_{1}, i_{2}, \ldots, i_{n}\right), \\
& h\left(i_{1}, \delta_{2}, \ldots, i_{n}\right)= h\left(i_{1}, \sigma_{2}+1, \ldots, i_{n}\right)-h\left(i_{1}, \sigma_{2}, \ldots, i_{n}\right), \\
& h\left(i_{1}, i_{2}, \ldots, \delta_{n}\right)= h\left(i_{1}, i_{2}, \ldots, \sigma_{n}+1\right)-h\left(i_{1}, i_{2}, \ldots, \sigma_{n}\right), \\
& d_{1}\left(\delta_{1}\right)= d_{1}\left(\sigma_{1}+1\right)-d_{1}\left(\sigma_{1}\right), \\
& d_{2}\left(\delta_{2}\right)=d_{2}\left(\sigma_{2}+1\right)-d_{2}\left(\sigma_{2}\right), \\
& d_{n}\left(\delta_{n}\right)=d_{n}\left(\sigma_{n}+1\right)-d_{n}\left(\sigma_{n}\right) .
\end{aligned}
$$

If $L_{g} h(x)=0$, then $\dot{y}=L_{f} h(x)$ is independent of $u$. We continue to calculate the second derivative of $y$, denoted by $y^{(2)}$ and then we obtain

$$
y^{(2)}=\frac{\partial L_{f} h}{\partial x}(f(x)+g(x) u)=L_{f}^{2} h(x)+L_{g} L_{f} h(x) u,
$$

where

$$
\begin{aligned}
& L_{f}^{2} h(x)=\frac{\partial L_{f} h}{\partial x_{1}} f_{1}+\cdots+\frac{\partial L_{f} h}{\partial x_{n}} f_{n}, \\
& L_{g} L_{f} h(x)=\frac{\partial L_{f} h}{\partial x_{1}} g_{1}+\cdots+\frac{\partial L_{f} h}{\partial x_{n}} g_{n}, \\
& \frac{\partial L_{f} h}{\partial x_{1}}=\sum_{i_{3}=\sigma_{3}}^{\sigma_{3}+1} \omega_{3}^{i_{3}} \cdots \sum_{i_{n}=\sigma_{n}}^{\sigma_{n}+1} \omega_{n}^{i_{n}} \frac{h\left(\delta_{1}, \delta_{2}, \ldots, i_{n}\right)}{d_{1}\left(\delta_{1}\right) d_{2}\left(\delta_{2}\right)} f_{2}+\cdots \\
& +\sum_{i_{2}=\sigma_{2}}^{\sigma_{2}+1} \omega_{2}^{i_{2}} \ldots \sum_{i_{n-1}=\sigma_{n-1}}^{\sigma_{n-1}+1} \omega_{n-1}^{i_{n-1}} \frac{h\left(\delta_{1}, i_{2}, \ldots, \delta_{n}\right)}{d_{1}\left(\delta_{1}\right) d_{n}\left(\delta_{n}\right)} f_{n} \\
& +\sum_{i_{2}=\sigma_{2}}^{\sigma_{2}+1} \omega_{2}^{i_{2}} \cdots \sum_{i_{n}=\sigma_{n}}^{\sigma_{n}+1} \omega_{n}^{i_{n}} \frac{h\left(\delta_{1}, i_{2}, \ldots, i_{n}\right)}{d_{1}\left(\delta_{1}\right)} \\
& \times \sum_{i_{2}=\sigma_{2}}^{\sigma_{2}+1} \omega_{2}^{i_{2}} \cdots \sum_{i_{n}=\sigma_{n}}^{\sigma_{n}+1} \omega_{n}^{i_{n}} \frac{f_{1}\left(\delta_{1}, i_{2}, \ldots, i_{n}\right)}{d_{1}\left(\delta_{1}\right)}+\cdots \\
& +\sum_{i_{1}=\sigma_{1}}^{\sigma_{1}+1} \omega_{1}^{i_{1}} \cdots \sum_{i_{n-1}=\sigma_{n-1}}^{\sigma_{n-1}+1} \omega_{n-1}^{i_{n-1}} \frac{h\left(i_{1}, i_{2}, \ldots, \delta_{n}\right)}{d_{n}\left(\delta_{n}\right)} \\
& \times \sum_{i_{2}=\sigma_{2}}^{\sigma_{2}+1} \omega_{2}^{i_{2}} \cdots \sum_{i_{n}=\sigma_{n}}^{\sigma_{n}+1} \omega_{n}^{i_{n}} \frac{f_{n}\left(\delta_{1}, i_{2}, \ldots, i_{n}\right)}{d_{1}\left(\delta_{1}\right)} \\
& \frac{\partial L_{f} h}{\partial x_{n}}=\sum_{i_{2}=\sigma_{2}}^{\sigma_{2}+1} \omega_{2}^{i_{2}} \ldots \sum_{i_{n}=\sigma_{n-1}}^{\sigma_{n-1}+1} \omega_{n-1}^{i_{n-1}} \frac{h\left(\delta_{1}, i_{2}, \ldots, \delta_{n}\right)}{d_{1}\left(\delta_{1}\right) d_{n}\left(\delta_{n}\right)} f_{1} \\
& +\cdots+\sum_{i_{1}=\sigma_{1}}^{\sigma_{1}+1} \omega_{1}^{i_{1}} \cdots \sum_{i_{n-2}=\sigma_{n-2}}^{\sigma_{n-2}+1} \omega_{n-2}^{i_{n-2}} \frac{h\left(i_{1}, \ldots, \delta_{n-1}, \delta_{n}\right)}{d_{n-1}\left(\delta_{n-1}\right) d_{n}\left(\delta_{n}\right)} f_{n-1} \\
& +\sum_{i_{2}=\sigma_{2}}^{\sigma_{2}+1} \omega_{2}^{i_{2}} \cdots \sum_{i_{n}=\sigma_{n}}^{\sigma_{n}+1} \omega_{n}^{i_{n}} \frac{h\left(\delta_{1}, i_{2}, \ldots, i_{n}\right)}{d_{1}\left(\delta_{1}\right)} \\
& \times \sum_{i_{1}=\sigma_{1}}^{\sigma_{1}+1} \omega_{1}^{i_{1}} \cdots \sum_{i_{n-1}=\sigma_{n-1}}^{\sigma_{n-1}+1} \omega_{n-1}^{i_{n-1}} \frac{f_{1}\left(i_{1}, i_{2}, \ldots, \delta_{n}\right)}{d_{n}\left(\delta_{n}\right)}+\cdots \\
& +\sum_{i_{1}=\sigma_{1}}^{\sigma_{1}+1} \omega_{1}^{i_{1}} \ldots \sum_{i_{n-1}=\sigma_{n-1}}^{\sigma_{n-1}+1} \omega_{n-1}^{i_{n-1}} \frac{h\left(i_{1}, i_{2}, \ldots, \delta_{n}\right)}{d_{n}\left(\delta_{n}\right)}
\end{aligned}
$$

$$
\times \sum_{i_{1}=\sigma_{1}}^{\sigma_{1}+1} \omega_{1}^{i_{1}} \ldots \sum_{i_{n-1}=\sigma_{n-1}}^{\sigma_{n-1}+1} \omega_{n-1}^{i_{n-1}} \frac{f_{n}\left(i_{1}, i_{2}, \ldots, \delta_{n}\right)}{d_{n}\left(\delta_{n}\right)}
$$

Once again, if $L_{g} L_{f} h(x)=0$, then $y^{(2)}=L_{f}^{2} h(x)$ is independent of $u$. Repeating this process, we see that if $h(x)$ satisfies

$$
\begin{gathered}
L_{g} L_{f}^{i} h(x)=0, \quad i=0,1, \ldots, \rho-2, \\
L_{g} L_{f}^{\rho-1} h(x) \neq 0
\end{gathered}
$$

then $u$ does not appear in the equations of $y$, $\dot{y}, \ldots, y^{(\rho-1)}$ and appears in the equation of $y^{(\rho)}$ with a nonzero coefficient:

$$
y^{(\rho)}=L_{f}^{\rho} h(x)+L_{g} L_{f}^{\rho-1} h(x) u .
$$

The foregoing equation shows clearly that the system is input-output linearizable, since the state feedback control

$$
u=\left(-L_{f}^{\rho} h(x)+v\right) / L_{g} L_{f}^{\rho-1} h(x)
$$

reduces the input-output map to $y^{(\rho)}=v$, which is a chain of $\rho$ integrators. In this case, the integer $\rho$ is called the relative degree of the system.

If $L_{g} L_{f}^{\rho-1} h\left(x_{t}\right)=0$, the relative degree cannot be defined at $x=x_{t}$. In some cases the relative degree can be defined at the point because we can adjust a partition of the state space for PB modeling so that $L_{g} L_{f}^{\rho-1} h\left(x_{t}\right) \neq 0$.

Definition 3.1: The nonlinear system is said to have relative degree $\rho, 1 \leq \rho \leq n$, in a region $D_{0} \subset D$ if

$$
\begin{aligned}
& L_{g} L_{f}^{i} h(x)=0, i=0,1, \cdots, \rho-2 \\
& L_{g} L_{f}^{\rho-1} h(x) \neq 0,
\end{aligned}
$$

for all $x \in D_{0}$..

The input-output linearized system can be formulated as

$$
\left\{\begin{array}{l}
\dot{\xi}=A \xi+B v \\
y=C \xi
\end{array}\right.
$$

where $\xi \in \mathfrak{R}^{\rho}, \quad C=(1,0, \ldots, 0,0)^{T}$,

$$
A=\left(\begin{array}{ccccc}
0 & 1 & 0 & \cdots & 0 \\
0 & 0 & 1 & \ddots & \vdots \\
\vdots & \vdots & \ddots & \ddots & 0 \\
0 & 0 & \cdots & 0 & 1 \\
0 & 0 & \cdots & 0 & 0
\end{array}\right), B=\left(\begin{array}{c}
0 \\
0 \\
\vdots \\
0 \\
1
\end{array}\right)
$$

Note that all the PB models (5) are transformed into the linear system (10). Therefore it is easy to design the stabilizing controller and analyze the stability of the PB systems.

According to the relative degree, three cases of linearized systems (10) must be considered.

Relative degree: $\rho=n$

In this case, the state vector of the input-output linearized 
system is

$$
z=\xi=\left(h(x), L_{f} h(x), \cdots, L_{f}^{\rho-1} h(x)\right)^{T}
$$

The state vector $z$ is necessary to be a diffeomorphism.

Relative degree: $\rho<n$

There is unobservable state ( $n-\rho$ dimensions). It is necessary to consider the zero dynamics of the unobservable state $\mu$. The state vector $z$ is necessary to be a diffeomorphism.

$$
\begin{aligned}
& z=(\xi, \mu)^{T}, \quad \xi \in \mathfrak{R}^{\rho}, \mu \in \mathfrak{R}^{n-\rho}, \\
& \dot{\mu}(\xi, \mu)=\zeta_{1}(\xi, \mu)+\zeta_{2}(\xi, \mu) v .
\end{aligned}
$$

$\dot{\mu}(0, \mu)$ is characterized by zero dynamics.

In the case of $L_{g} L_{f}^{i} h(x)=0, \forall i$, the proposed approach cannot be applied.

When the relative degree $\rho \leq n$, the input-output linearizing controller is $u=\alpha(x)+\beta(x) v$, where

$$
\alpha(x)=-L_{f}^{\rho} h(x) / L_{g} L_{f}^{\rho-1} h(x), \beta(x)=1 / L_{g} L_{f}^{\rho-1} h(x) .
$$

In the following, we assume the relative degree is $n$ (full). The stabilizing linear controller $v=-F \xi$ of the linearized system (10) can be obtained so that the transfer function $G=C(s I-A)^{-1} B$ is Hurwitz.

The linearizing controller can be characterized as the LUT (Look-Up-Table) controller, where the LUT-controller is widely used for industrial applications, in particular, for vehicle control because of simplicity and also visibility as a nonlinear controller. In the case of the LUT-controller, control inputs are calculated by interpolation based on the table. When bilinear piecewise interpolation is adopted, the LUT-controller is found to be exactly the PB system.

\section{OBSERVER-BASED PB CONTROLLER}

We propose an observer-based PB controller to estimate the coordinate transformation $z$ by using the error of $y-\hat{y}$. In this paper, we construct a full-order state observer for PB control system as shown in Fig. 2. In this figure, $T$ and $1 / S$ show the coordinate transformation and the integrator. $F$ is the feedback gain, $K$ is the observer gains, and $r(\dot{r}=0)$ is the set point signal. Due to lack of space, we only discuss the nonlinear system with the relative degree $\rho=n$. The following approach can be also applied to the nonlinear systems with $\rho<n$. We consider the linearized system using PB models.

$$
\left\{\begin{array}{l}
\dot{z}=A z+B v \\
y=C z
\end{array}\right.
$$

The full-order state observer system is of a following form.

$$
\left\{\begin{array}{l}
\dot{\hat{z}}=A \hat{z}+B v+K(y-\hat{y}), \\
\hat{y}=C \hat{z},
\end{array}\right.
$$

where the controller

$$
v=-F \hat{z} \text {. }
$$

The closed loop system including the systems (12) and (13) is obtained as

$$
\left(\begin{array}{c}
\dot{z} \\
\dot{\hat{z}}
\end{array}\right)=\left(\begin{array}{cc}
A & -B F \\
K C & A-B F-K C
\end{array}\right)\left(\begin{array}{l}
z \\
\hat{z}
\end{array}\right)
$$

The following closed loop system can be obtained by using a coordinate transformation $e=\hat{z}-z$ and the similarity transformation.

$$
\left(\begin{array}{c}
\dot{z} \\
\dot{e}
\end{array}\right)=\left(\begin{array}{cc}
A-B F & -B F \\
0 & A-K C
\end{array}\right)\left(\begin{array}{l}
z \\
e
\end{array}\right)
$$

We can design the feedback gain $F$ and observer gain $K$ separately. This is based on the separation principle. In general, one selects the poles of $A-K C$ on the left side of the poles of $A-B F$. Finally, the observer-based PB controller is obtain as

$$
u=\alpha(x)+\beta(x) v=\frac{-L_{f}^{\rho} h(x)-F \hat{z}}{L_{g} L_{f}^{\rho-1} h(x)}
$$

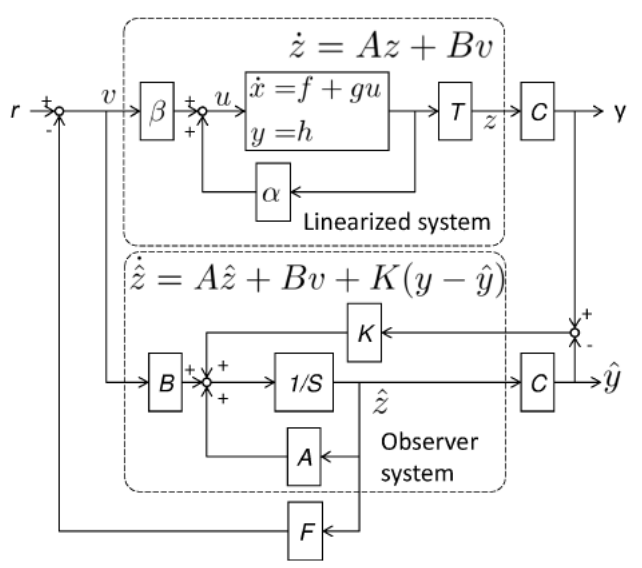

Fig. 2. Full-order state observer of PB control system.

\section{NUMERICAL EXAMPLES}

Consider the nonlinear system

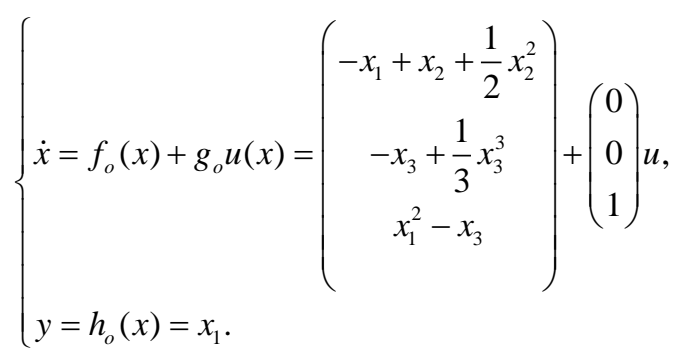

The conventional I/O feedback linearization controller is designed as

$$
u(x)=\frac{-L_{f_{o}}^{3} h_{o}(x)}{L_{g_{o}} L_{f_{o}}^{2} h_{o}(x)}+\frac{1}{L_{g_{o}} L_{f_{o}}^{2} h_{o}(x)} v,
$$


where

$$
\begin{aligned}
L_{f_{o}}^{3} h_{o}(x) & =f_{o_{1}}(x)-\left(1+x_{2}\right) f_{o_{2}}(x) \\
& +\left(1+x_{2}\right)\left(-1+x_{3}^{2}\right) f_{o_{3}}(x), \\
L_{g_{o}} L_{f_{o}}^{2} h_{o}(x) & =\left(1+x_{2}\right)\left(-1+x_{3}^{2}\right), \\
f_{o}(x) & =\left(f_{o_{1}}(x), f_{o_{2}}(x), f_{o_{3}}(x)\right)^{T} .
\end{aligned}
$$

The linearized controller cannot be applied to stabilize the nonlinear system (15) at $x_{2}=-1$ and $x_{3}= \pm 1$ since $L_{g_{o}} L_{f_{o}}^{2} h_{o}(x)=0$ at these points. Therefore, the linearizable region is restricted to $x_{2} \in[-1, \infty)$ and $x_{3} \in[-1,1]$. Divide the state space of the nonlinear system (15) as $x_{1}=\{-3,-1.5, \cdots, 3\} \quad, \quad x_{2}=\{-2,-1.2,0,1.2,2\} \quad$ and $x_{3}=\{-2,-1.2,0,1.2,2\}$, then the PB model is constructed as

$$
\left\{\begin{array}{l}
\dot{x}=f(x)+g u(x)=\left(f_{1}(x), f_{2}(x), f_{3}(x)\right)^{T}+\left(\begin{array}{l}
0 \\
1
\end{array}\right) u, \\
y=h(x)=x_{1}
\end{array}\right.
$$

where

$$
\begin{aligned}
f_{1}(x) & =\sum_{i=\sigma}^{\sigma+1} \sum_{j=\tau}^{\tau+1} \omega_{1}^{i}\left(x_{1}\right) \omega_{2}^{j}\left(x_{2}\right) f_{1}(i, j, \cdot), \\
f_{2}(x) & =\sum_{k=v}^{v+1} \omega_{3}^{k}\left(x_{3}\right) f_{2}(\cdot, \cdot, k), \\
f_{3}(x) & =\sum^{\sigma+1} \sum^{v+1} \omega_{1}^{i}\left(x_{1}\right) \omega_{3}^{k}\left(x_{3}\right) f_{3}(i, \cdot, k) .
\end{aligned}
$$

Here, $f_{1}(i, j, \cdot)$ means that it is independent of $k$ and also about $f_{2}(\cdot, \cdot, k)$ and $f_{3}(i, \cdot, k)$.

TABLE I: PB MODELS

\begin{tabular}{|c||c|c|c|c|c|}
\hline$f_{1}\left(\dot{s}_{2}, \cdot\right)$ & $d_{1}(-2)$ & $d_{1}(-1)$ & $d_{1}(0)$ & $d_{1}(1)$ & $d_{1}(2)$ \\
\hline \hline$d_{2}(-2)$ & 3.00 & 1.50 & 0 & -1.50 & -3.00 \\
\hline$d_{2}(-1)$ & 2.52 & 1.02 & -0.48 & -1.98 & -3.48 \\
\hline$d_{2}(0)$ & 3.00 & 1.50 & 0 & -1.50 & -3.00 \\
\hline$d_{2}(1)$ & 4.92 & 3.42 & 1.92 & 0.42 & -1.08 \\
\hline$d_{2}(2)$ & 7.00 & 5.50 & 4.00 & 2.50 & 1.00 \\
\hline \hline$f_{2}\left(\dot{s}_{;} ; k\right)$ & $d_{1}(-2)$ & $d_{1}(-1)$ & $d_{1}(0)$ & $d_{1}(1)$ & $d_{1}(2)$ \\
\hline \hline$d_{3}(-2)$ & -0.667 & -0.667 & -0.667 & -0.667 & -0.667 \\
\hline$d_{3}(-1)$ & 0.624 & 0.624 & 0.624 & 0.624 & 0.624 \\
\hline$d_{3}(0)$ & 0 & 0 & 0 & 0 & 0 \\
\hline$d_{3}(1)$ & -0.624 & -0.624 & -0.624 & -0.624 & -0.624 \\
\hline$d_{3}(2)$ & 0.667 & 0.667 & 0.667 & 0.667 & 0.667 \\
\hline \hline$f_{3}\left(\hat{s}_{3} ; k\right)$ & $d_{1}(-2)$ & $d_{1}(-1)$ & $d_{1}(0)$ & $d_{1}(1)$ & $d_{1}(2)$ \\
\hline \hline$d_{3}(-2)$ & 11.0 & 4.25 & 2.00 & 4.25 & 11.0 \\
\hline$d_{3}(-1)$ & 10.2 & 3.45 & 1.20 & 3.45 & 10.2 \\
\hline$d_{3}(0)$ & 9.00 & 2.25 & 0 & 2.25 & 9.00 \\
\hline$d_{3}(1)$ & 7.80 & 1.05 & -1.20 & 1.05 & 7.80 \\
\hline$d_{3}(2)$ & 7.00 & 0.25 & -2.00 & 0.25 & 7.00 \\
\hline
\end{tabular}

Table I shows the PB models of $f_{1}(x), f_{2}(x)$ and $f_{3}(x)$. We design the stabilizing controller:

$$
u(x)=\frac{-L_{f}^{3} h(x)}{L_{g} L_{f}^{2} h(x)}+\frac{1}{L_{g} L_{f}^{2} h(x)} v
$$

where

$$
\begin{aligned}
& v=-F z=-(2.12,5.27,2.79) z, \\
& z=\left(x_{1}, f_{1}(x), \frac{\partial f_{1}(x)}{\partial x_{1}} f_{1}(x)+\frac{\partial f_{1}(x)}{\partial x_{2}} f_{2}(x)\right)^{T},
\end{aligned}
$$

$$
\begin{aligned}
L_{f}^{3} h(x)= & \left(\frac{\partial f_{1}(x)}{\partial x_{1}}\right)^{2} f_{1}(x) \\
& +\frac{\partial f_{1}(x)}{\partial x_{1}} \frac{\partial f_{1}(x)}{\partial x_{2}} f_{2}(x)+\frac{\partial f_{1}(x)}{\partial x_{2}} \frac{\partial f_{2}(x)}{\partial x_{3}} f_{3}(x), \\
L_{g} L_{f}^{2} h(x)= & \frac{\partial f_{1}(x)}{\partial x_{2}} \frac{\partial f_{2}(x)}{\partial x_{3}}, \\
\frac{\partial f_{1}(x)}{\partial x_{1}}= & \left(f_{1}(\sigma+1, \cdot, \cdot)-f_{1}(\sigma, \cdot, \cdot)\right) / d_{1}\left(\Delta_{\sigma}\right), \\
\frac{\partial f_{1}(x)}{\partial x_{2}} & =\left(f_{1}(\cdot, \tau+1, \cdot)-f_{1}(\cdot, \tau, \cdot)\right) / d_{2}\left(\Delta_{\tau}\right), \\
\frac{\partial f_{2}(x)}{\partial x_{3}} & =\left(f_{2}(\cdot, \cdot, v+1)-f_{2}(\cdot, \cdot, v)\right) / d_{3}\left(\Delta_{v}\right) .
\end{aligned}
$$

Note that $\partial f_{1}\left(x_{1}\right) / \partial x_{1}, \partial f_{1}\left(x_{1}\right) / \partial x_{2}$ and $\partial f_{2}\left(x_{1}\right) / \partial x_{2}$ in the stabilizing controller (16) are constant parameters. The stabilizing controller is represented as a bilinear function. Therefore the controller is characterized as the LUT controller.

From the original $f_{1}(x)$ and $f_{2}(x)$, we can derive

$$
\begin{aligned}
& f_{1}(\cdot, \tau+1, \cdot)-f_{1}(\cdot, \tau, \cdot) \\
= & {\left[1+1 / 2\left(d_{2}(\tau)+d_{2}(\tau+1)\right)\right]\left(d_{2}(\tau+1)-d_{2}(\tau)\right), } \\
& f_{2}(\cdot, \cdot, v+1)-f_{2}(\cdot, \cdot, v) \\
= & {\left[-1+1 / 3\left(d_{3}(v)^{2}+d_{3}(v) d_{3}(v+1)+d_{3}(v+1)^{2}\right)\right] } \\
& \times\left(d_{3}(v+1)-d_{3}(v)\right) .
\end{aligned}
$$

If $\quad 1+1 / 2\left(d_{2}(\tau)+d_{2}(\tau+1)\right) \neq 0 \quad$ and $-1+1 / 3\left(d_{3}(v)^{2}+d_{3}(v) d_{3}(v+1)+d_{3}(v+1)^{2}\right) \neq 0, \quad$ then $L_{g} L_{f}^{2} h(x) \neq 0$. These conditions are satisfied by the current partition of the state space. Therefore, the PB model is found to be globally feedback linearizable. Fig. 3 shows a simulation result of the initial condition $x(0)=(1,1.5,1)^{T}$, where it is seen that $x_{3}$ exceeds 1 : note that the original linearization region is $-1<x_{3}<1$.

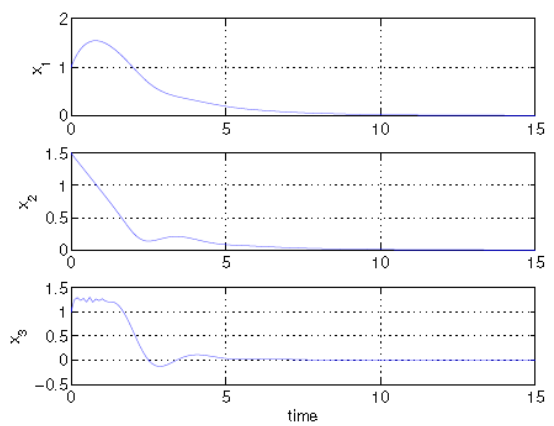

Fig. 3. Control responses using the PB controller

We apply the observer design method to this nonlinear system. We select the poles of $A-K C$ on the left side of the poles of $A-B F$, then the feedback gain is calculated as $F=(2.12,5.27,2.79)$ and observer gain is calculated as $K=(33.0,362,1320)^{T}$. Fig. 4 shows a simulation result of the initial condition $x(0)=(1,1.5,1)^{T}$, where it is also seen 
that $x_{3}$ exceeds 1 : note that the original linearization region is $-1<x_{3}<1$. Fig. 5 shows the estimated state trajectories of $\mathrm{PB}$ control with the state observer.

Note that the observer-based PB controller is simpler than the conventional feedback linearization one. Since the nonlinear terms of the controller (16) are not the original nonlinear terms (e.g., $x_{1}^{2}, x_{2}^{2}$ and $x_{3}^{3}$ ) and the PB controller is represented as an LUT one. However the control performance based on PB model is the same as the conventional one.
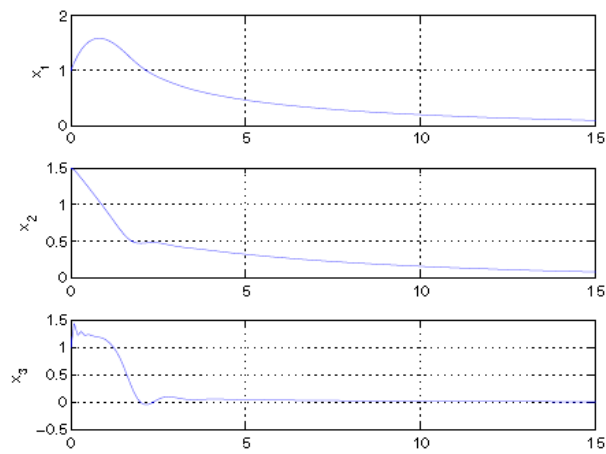

Fig. 4. State responses using the observer-based PB controller.
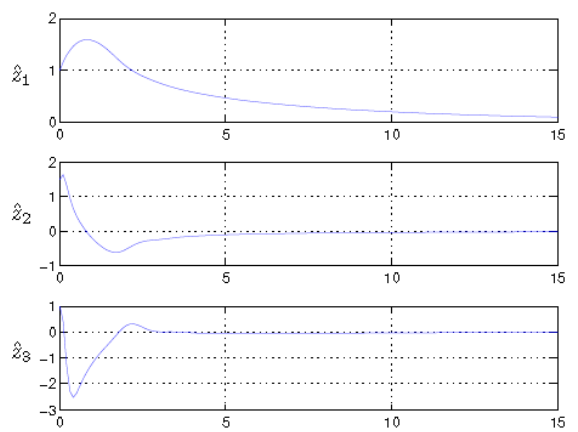

Fig. 5. Estimated state trajectories using the observer-based PB controller.

\section{CONCLUSION}

This paper has proposed a full-order state observer design of nonlinear control systems approximated by piecewise bilinear (PB) models. We have constructed PB model of nonlinear control systems. The approximated system is found to be fully parametric. The input-output (I/O) feedback linearization has been applied to stabilize PB control systems. The design method is capable of designing the state observer and the servo controller of nonlinear systems separately. Although the PB controller is simpler than the conventional I/O feedback linearization controller, the control performance based on PB model is the same as the conventional one. We have presented the PB modeling combined with the conventional feedback linearization as a very powerful tool for the analysis and synthesis of nonlinear control systems. An illustrative example has confirmed the feasibility of our proposals.

\section{ACKNOWLEDGMENT}

The authors would like to thank Dr. Dimitar Filev and Dr. Yan Wang of Ford Motor Company for his valuable comments and discussions. This work was supported by a
URP grant from Ford Motor Company which the authors thankfully acknowledge. In addition, this work was supported by Grant-in-Aid for Young Scientists (B: 23700276) of The Ministry of Education, Culture, Sports, Science and Technology in Japan.

\section{REFERENCES}

[1] E. D. Sontag, "Nonlinear regulation: the piecewise linear approach," IEEE Trans. Autom. Control, vol. 26, pp. 346-357, 1981.

[2] M. Johansson and A. Rantzer, "Computation of piecewise quadratic lyapunov functions of hybrid systems," IEEE Trans. Autom. Control, vol. 43, no. 4, pp. 555-559, 1998.

[3] J. Imura and A. van der Schaft, "Characterization of well-posedness of piecewise-linear systems," IEEE Trans. Autom. Control, vol. 45, pp. 1600-1619, 2000.

[4] G. Feng, G. P. Lu, and S. S. Zhou, "An approach to hinfinity controller synthesis of piecewise linear systems," Communications in Information and Systems, vol. 2, no. 3, pp. 245-254, 2002.

[5] M. Sugeno, "On stability of fuzzy systems expressed by fuzzy rules with singleton consequents," IEEE Trans. Fuzzy Syst., vol. 7, no. 2, pp.201-224, 1999.

[6] M. Sugeno and T. Taniguchi, "On improvement of stability conditions for continuous mamdani-like fuzzy systems," IEEE Tran. Systems, Man, and Cybernetics, vol. 34, no. 1, pp. 120-131, 2004.

[7] T. Taniguchi and M. Sugeno, "Stabilization of nonlinear systems based on piecewise lyapunov functions," in FUZZ-IEEE 2004, 2004, pp. 1607-1612.

[8] "Piecewise bilinear system control based on full-state feedback linearization," in SCIS \& ISIS 2010, 2010, pp. 1591-1596.

[9] "Stabilization of nonlinear systems with piecewise bilinear models derived from fuzzy if-then rules with singletons," in FUZZ-IEEE 2010, 2010, pp. 2926-2931.

[10] T. Taniguchi, L. Eciolaza, and M. Sugeno, "LUT controller design with piecewise bilinear systems using estimation of bounds for approximation errors," Journal of Advanced Computational Intelligence and Intelligent Informatics, vol. 17, no. 6, 2013.

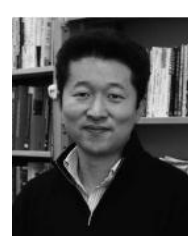

Tadanari Taniguchi received the B.S. and M.S degrees in mechanical systems engineering from Kanazawa University, Kanazawa, Japan, in 1996 and 1998 respectively, and the Ph.D. degree in mechanical and control engineering from University of electro-communications, Tokyo, Japan, in 2001. From 2001 to 2005 , he was a research scientist of the brain science institute, RIKEN, Saitama, Japan. Since 2005, he has been with IT education center, Tokai University, Kanagawa, Japan. $\mathrm{He}$ is currently an associate professor with Tokai University. His research interests include intelligent control, nonlinear control, feedback error learning and expert system.

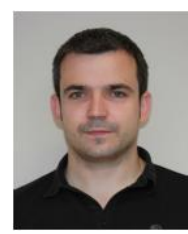

Luka Eciolaza received the M.S. degree in mondragon sistemas de informacion, Spain and the Ph.D. degree in University of Sheffield, UK, in 2004 and 2007, respectively. In 2007 to 2010, He was with rolls-royce PLC, Derby, UK. Since 2010, he has been a postdoctoral researcher with european centre for soft computing, Spain.

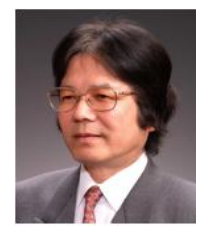

Michio Sugeno graduated from the Department of Physics, The University of Tokyo, He worked at mitsubishi atomic power industry. T hen, he served the Tokyo institute of technology as research associate, associate professor and professor from 1965 to 2000 . After retiring from the Tokyo Institute of Technology, he worked as laboratory head at the Brain Science Institute, RIKEN from 2000 to 2005 , and then, as distinguished visiting professor at Doshisha University from 2005 to 2010. $\mathrm{He}$ is currently emeritus professor at the Tokyo institute of technology, Japan, and emeritus researcher at the European Centre for soft computing, Spain. He was as president of the Japan society for fuzzy theory and systems from 1991 to 1993, and also president of the international fuzzy systems association from 1997 to 1999 . He is the first recipient of the IEEE Pioneer. Award in Fuzzy Systems with Zadeh in 2000. He also received the 2010 IEEE Frank Rosenblatt Award and recently Kampt de Ferit Award in 2012 